\title{
First-Order Modal Logic in the Necessary Framework of Objects*
}

\author{
Peter Fritz ${ }^{\dagger}$ \\ Final draft
}

\begin{abstract}
I consider the first-order modal logic which counts as valid those sentences which are true on every interpretation of the non-logical constants. Based on the assumptions that it is necessary what individuals there are and that it is necessary which propositions are necessary, Timothy Williamson has tentatively suggested an argument for the claim that this logic is determined by a possible world structure consisting of an infinite set of individuals and an infinite set of worlds. He notes that only the cardinalities of these sets matters, and that not all pairs of infinite sets determine the same logic. I use so-called two-cardinal theorems from model theory to investigate the space of logics and consequence relations determined by pairs of infinite sets, and show how to eliminate the assumption that worlds are individuals from Williamson's argument.
\end{abstract}

\section{Introduction}

Modal logic is a branch of formal logic with a wide variety of applications in philosophy, computer science, linguistics, economics, and other fields. In Modal Logic as Metaphysics (2013), Timothy Williamson applies first-order modal logic as well as some of its higher-order extensions to metaphysics. One of the primary roles which modal logic serves in this book is as a regimented language in which various metaphysical theses are formulated. This use is best illustrated with an example.

The overall aim of Williamson's book is to defend necessitism, the view, roughly, that it is necessary what there is. More explicitly, one might describe it as the view that nothing could have failed to be anything, and that this is necessarily so, i.e., as the view that necessarily, everything is necessarily something. For various reasons, such as to rule out ambiguities, it can be helpful to formulate such views in a formal language. Williamson formulates necessitism in first-order modal logic as follows:

(NNE) $\square \forall x \square \exists y x=y$

* Forthcoming in the Canadian Journal of Philosophy. The final publication is available at http://www.tandfonline.com/doi/full/10.1080/00455091.2015.1132976.

$\dagger$ Thanks to Jeremy Goodman and Timothy Williamson for comments on a draft, Øystein Linnebo, Beau Mount and Sam Roberts for discussions of Kreisel's Principle, and audiences in Cambridge, Oxford, Paris and Montreal - especially my commentators, Michael Gabbay in Cambridge and Beau Mount in Oxford - for questions and comments. 
The role of the formula NNE in Williamson's discussion is not just as an aid to understanding the natural language statement of necessitism given before, e.g., by indicating how to resolve potential scope ambiguities. Rather, NNE on its own is intended to be a statement of the view of necessitism. So, in addition to having formal properties such as being derivable in certain calculi but not others, and being valid on certain model theories but not others, NNE also needs to be endowed with meaning just as the English sentences of this article are endowed with meaning. Williamson's use of NNE therefore presupposes an intended interpretation of the formal language of first-order modal logic. In keeping with the variety of uses to which modal logics can be put, this need not be distinguished among various other possible interpretations of the language; it must only be isolated for the particular discussion in Williamson's book.

Williamson specifies his intended interpretation of first-order modal logic by saying that he intends quantifiers to be read unrestrictedly, and modal operators as expressing metaphysical necessity and possibility; he assumes that the intended interpretation of the identity connective and the Boolean operators is clear. This stipulation is of course not uncontroversial, as the intelligibility of both unrestricted quantification and the metaphysical interpretation of modal expressions have been questioned. Even more controversially, Williamson extends his intended interpretation to higher-order modal logic by claiming that there is a way of interpreting higher-order quantifiers analogously to the unrestricted interpretation of first-order quantifiers. This interpretation is neither to be understood in terms of our informal talk of properties and relations nor in terms of any set-theoretic model theory. For the purposes of this article, it will be assumed that with these stipulations, Williamson has succeeded in singling out an intended interpretation for first- and higher-order modal logic; for further discussion, see Williamson (2003). In the following, talk of properties and relations will sometimes be used as informal glosses of what should properly be formulated in higher-order logic; the term individual will be used to indicate first-order quantification. Thus, sets will be understood to be individuals. This is worth noting since in impure set theory, the term individual is sometimes used for non-sets (also called urelemente).

The intended interpretation described so far does not provide any interpretation of non-logical constants. So, while closed formulas without non-logical constants, such as NNE, are given an intended interpretation, closed formulas with non-logical constants are only partially interpreted. However, such formulas at least receive an intended interpretation relative to an interpretation of the non-logical constants occurring in it. (As noted below, quantification over such interpretations can be understood in terms of higher-order quantification.) Consequently, the intended interpretation also determines which formulas are true on every interpretation of the non-logical constants (and the intended interpretation of the logical constants). Williamson calls these formulas metaphysically universal. Let $\Lambda_{\mathrm{MU}}$ be the set of metaphysically universal formulas of firstorder modal logic. (The formal language will be specified more precisely below). Analogously, let $\vDash_{\mathrm{MU}}$ be the relation which relates a set of closed formulas of first-order modal logic to a single such formula just in case the latter is true on any interpretation of non-logical constants on which all members of the former are true; of course, this closely corresponds what Tarski (2002 [1936]) calls the relation of following logically. Whatever one calls $\Lambda_{\mathrm{MU}}$ and $\vDash_{\mathrm{MU}}$, Williamson (2013, p. 92) observes that their investigation has to be carried out. 
Williamson (2013, p. 145) tentatively suggests an argument for the claim that $\Lambda_{\mathrm{MU}}$ is determined by a certain kind of Kripke frame for first-order modal logic consisting of two infinite sets. In the usual terminology, one of these sets is the domain of quantification and the other the set of worlds. The frames have no varying domain function since Williamson holds that it is a necessary matter what individuals there are; similarly, they have no accessibility relation since he holds that it is a necessary matter whether a given proposition is necessary. (Williamson's endorsement of the latter is in fact rather tentative, but since this won't be criticized in the following, there is no harm in exploring a position which might be somewhat stronger than Williamson's own.) Such a frame determines a set of closed formulas of first-order modal logic as the set of such formulas which are true in every world of every model based on it, on the usual definitions of model and truth (defined more explicitly below). It is easy to see that only the cardinalities of the two sets making up a given frame matter for which set of formulas it determines. Thus, any two infinite cardinals $\kappa, \lambda$ can be understood as directly determining a set of closed formulas of first-order modal logic; call it $\Lambda_{\kappa, \lambda}$. Here, $\kappa$ indicates the cardinality of the domain of quantification and $\lambda$ the cardinality of the set of worlds; we might therefore read " $\Lambda_{\kappa, \lambda}$ " as "the logic of $\kappa$ individuals and $\lambda$ worlds". Williamson's argument aims to establish that $\Lambda_{\mathrm{MU}}=\Lambda_{\kappa, \lambda}$ for some infinite cardinals $\kappa$ and $\lambda$. The analogous definition of a consequence relation $\vDash_{\kappa, \lambda}$ raises the question whether $\vDash_{\mathrm{MU}}=\vDash_{\kappa, \lambda}$ for some infinite cardinals $\kappa$ and $\lambda$.

As Williamson (2013, p. 144) notes, one might conjecture that $\Lambda_{\mathrm{MU}}$ is uniquely determined by the claim that $\Lambda_{\mathrm{MU}}=\Lambda_{\kappa, \lambda}$ for some infinite cardinals $\kappa$ and $\lambda$, using some modal analog of the Löwenheim-Skolem theorem. And indeed, an analog of the downward Löwenheim-Skolem theorem was already proven in Kripke $(1959$, p. 7$)$, from which it follows that $\Lambda_{\aleph_{0}, \aleph_{0}} \subseteq \Lambda_{\kappa, \lambda}$. However, Kripke's result does not establish the converse containment. In fact, as Williamson points out, this is not the case, as $\forall x \diamond \forall y(U y \leftrightarrow x=y)$ (which might be read as saying that every individual could be the unique $U$ ) can only be verified by a model based on a frame in which there are at least as many worlds as individuals, and so that the negation of this formula is a member of $\Lambda_{\kappa, \lambda}$ just in case $\kappa>\lambda$. On the one hand, this shows that $\Lambda_{\kappa, \lambda}$ and $\vDash_{\kappa, \lambda}$ depend on the choice of $\kappa$ and $\lambda$; on the other hand, it is clear by cardinality considerations that many pairs of infinite cardinals $\kappa, \lambda$ determine the same sets $\Lambda_{\kappa, \lambda}$ and $\vDash_{\kappa, \lambda}$.

How exactly do $\Lambda_{\kappa, \lambda}$ and $\vDash_{\kappa, \lambda}$ depend on $\kappa$ and $\lambda$ ? This is the question to be investigated in section 2 . It turns out that $\Lambda_{\kappa, \lambda}$ and $\vDash_{\kappa, \lambda}$ are uniquely determined by the condition that $\lambda \geq \kappa$, but sensitive to the cardinalities of $\lambda$ and $\kappa$ if $\lambda<\kappa$, with the different pairs of infinite cardinals determining infinitely many different logics. Furthermore, in some instances, whether two such pairs determine the same logic depends on set-theoretic principles which are likely not to be settled by first-order ZFC. This section is of independent interest; as will become clear, it could be reformulated in terms of extensions of the standard Löwenheim-Skolem theorem to first-order modal logic on the present model theory. Section 3 returns to Williamson's argument that $\Lambda_{\mathrm{MU}}$ is given by a pair of infinite cardinals. Some difficulties in evaluating one of the argument's premises will be raised, and it will be noted that the argument relies on worlds being individuals. The main contribution of this section is to show how to dispense with this last assumption, independently of any particular 
understanding of possible worlds.

Metaphysical universality is a simple and natural understanding of logical validity and consequence. In the case of non-modal first-order logic, there are well-developed arguments which uniquely determine the set of metaphysically universal sentences, namely those derivable in a standard calculus extended by an axiom schema which says that there are at least $n$ individuals, for each $n<\omega$ (Williamson, 2000a, Rayo and Williamson, 2003, Friedman, 1999); modeltheoretically, this is the set of sentences valid on any fixed infinite set, or the class of all infinite sets. Williamson's necessary framework of objects (Williamson, 2000b, 2013, section 8.1), according to which it is necessary what individuals there are as well as necessary which propositions are necessary, is the strongest and simplest non-trivial theory of modal metaphysics available. The results below show that even on this assumption, the addition of necessity to first-order logic turns determining the set of metaphysically universal sentences into an extremely difficult issue: doing so involves not only settling difficult questions in metaphysics, concerning the relative numbers of worlds and individuals, but potentially also difficult questions in set theory or higher-order logic. In general, such questions only become harder if the necessary framework of objects is rejected (although particular metaphysical assumptions might of course settle the matter). On the present understanding of logical validity and consequence, firstorder modal logic is therefore far less well understood than first-order non-modal logic.

\section{Set-Theoretic Models}

To specify the formal languages under consideration more precisely, fix a countably infinite set $V$ of individual variables and a relational signature $\sigma$ containing a countable infinity of relation symbols for each finite arity. Let $\mathcal{L}_{\square}$ be a standard first-order modal language with identity based on $V$ and $\sigma$. As explained above, it will be assumed that there is an intended interpretation of this language, on which quantifiers are interpreted unrestrictedly, and $\square$ as expressing

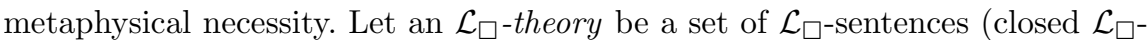
formulas), and an $\mathcal{L}_{\square}$-sequent a tuple $\langle\Gamma, \varphi\rangle$ consisting of an $\mathcal{L}_{\square \text {-theory } \Gamma \text { and }}$

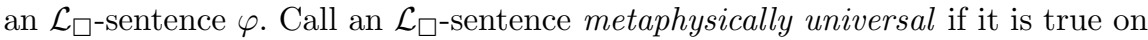
every interpretation assigning an $n$-ary relation to each $n$-ary relation symbol in $\sigma$; call an $\mathcal{L}_{\square \text {-sequent }}\langle\Gamma, \varphi\rangle$ metaphysically universal if on every such interpretation, all elements of $\Gamma$ are true only if $\varphi$ is true. Let $\Lambda_{\mathrm{MU}}$ be the set of metaphysically universal $\mathcal{L}_{\square}$-sentences and $\vDash_{\mathrm{MU}}$ the set of metaphysically universal $\mathcal{L}_{\square \text {-sequents. }}$

The notion of an interpretation of the relation symbols of $\sigma$ appealed to in the definition of metaphysical universality is informal, but - it will be assumed in the following - nonetheless rigorous. The definition can be formalized in a higher-order metalanguage, along the lines of Williamson (2013, section 5.7), and this can be carried over to the extension of $\mathcal{L}_{\square}$ by second-order quantifiers, interpreting such quantifiers homophonically. Since the relevant metalanguage is always of a higher order than the object language under consideration, the assumption that the resulting truth predicates are transparent does not conflict with Tarksi's undefinability theorem.

Assume the truth of first-order ZFC (interpreting quantifiers as implicitly 
restricted to the pure sets) and the von Neumann construction of ordinals, on which every ordinal is the set of ordinals smaller than it. Let a frame be a pair $\mathfrak{F}=\langle D, W\rangle$ of non-empty (pure) sets; informally, $D$ and $W$ will be called the sets of "individuals" and "worlds", respectively. Let a Kripke model based on $\mathfrak{F}$ be a tuple $\mathfrak{M}=\langle D, W, i$, @ $\rangle$, where $i$ is a function mapping each $n$-ary relation symbol in $\sigma$ to a function mapping each $w \in W$ to a set $i(R)(w)$ of $n$-tuples on $D$, and @ $\in W$; informally, $i$ and @ will be called the "interpretation function" and the "actual world", respectively. As usual, the interpretation of unary relations will be simplified by identifying any individual with its singleton sequence. Truth of an $\mathcal{L}_{\square}$-formula $\varphi$ in $\mathfrak{M}$ is defined as usual relative to an element $w \in W$ and an assignment function $a: V \rightarrow D$, written $\mathfrak{M}, w, a \vDash \varphi$. (Note that frames do not contain an accessibility relation, so $\square \varphi$ is true in a world if $\varphi$ is true in every world; similarly, there is no relativization of the domain of first-order quantifiers to worlds.) From this, truth of a sentence $\varphi$ in $\mathfrak{M}$ is derived as $\mathfrak{M}$, @, $a \vDash \varphi$ for all assignments $a$, and written $\mathfrak{M} \vDash \varphi$. A Kripke model of an $\mathcal{L}_{\square}$-theory $\Gamma$ is a Kripke model in which all members of $\Gamma$ are true. $\Gamma$ is satisfiable on a frame $\mathfrak{F}$ if there is a Kripke model of $\Gamma$ based on $\mathfrak{F}$. Here and in the following, any notion - such as being satisfiable - is extended from theories to sentences, treating a sentence as its singleton.

A frame $\mathfrak{F}$ naturally determines a logic (a set of sentences) and a consequence relation (a set of sequents), by counting a sentence as valid if it is true in all models based on $\mathfrak{F}$, and a sequent as valid if its conclusion is true in all models based on $\mathfrak{F}$ in which all of its premises are true. Any frames with the same numbers of individuals and worlds will determine the same logic and the same consequence relation, so define, for any cardinals $\kappa$ and $\lambda, \vDash_{\kappa, \lambda}$ to be the set of $\mathcal{L}_{\square \text {-sequents }}\langle\Gamma, \varphi\rangle$ such that $\mathfrak{M} \vDash \varphi$ for all Kripke models $\mathfrak{M}$ of $\Gamma$ on $\langle\kappa, \lambda\rangle$,

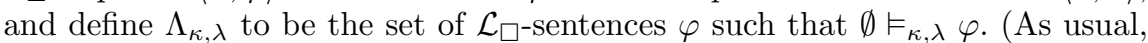
cardinals are taken to be initial ordinals.) Thus $\Lambda_{\kappa, \lambda}$ and $\vDash_{\kappa, \lambda}$ can informally be thought of as the logic and consequence relation of frames with $\kappa$ individuals and $\lambda$ worlds.

\section{$2.1 \quad$ Reduction}

To investigate the space of logics and consequence relations $\Lambda_{\kappa, \lambda}$ and $\vDash_{\kappa, \lambda}$ for infinite cardinals $\kappa$ and $\lambda$, membership in $\Lambda_{\kappa, \lambda}$ and $\vDash_{\kappa, \lambda}$ will first be reduced to the so-called two-cardinal problem in model theory. As the two-cardinal problem has been investigated extensively, this relatively simple reduction immediately gives rise to a number of corollaries which would be extremely difficult to prove directly. Such corollaries will then be used to lay out the basic features of the space of logics and consequence relations determined by pairs of infinite cardinals, including results on compactness and recursive enumerability.

Let $\mathcal{L}$ be the language of (non-modal) first-order logic with identity based on $\sigma$ and $V$. Let a model be a tuple $\mathfrak{A}=\langle D, e\rangle$ where $D$ is a non-empty set and $e$ is a function which maps every $n$-ary relation symbol $R$ of $\sigma$ to a set $e(R)$ of $n$-tuples on $D$; informally, $D$ and $e$ will be called the set of "individuals" and the "interpretation function", respectively. Truth of an $\mathcal{L}$-formula $\varphi$ in $\mathfrak{A}$ is defined as usual relative to an assignment function $a: V \rightarrow D$, written $\mathfrak{A}, a \vDash \varphi$. From this, truth of a sentence $\varphi$ in $\mathfrak{A}$ is derived as $\mathfrak{A}, a \vDash \varphi$ for all assignments $a$, and written $\mathfrak{A} \vDash \varphi$. An $\mathcal{L}$-theory is a set of sentences of $\mathcal{L}$; an $\mathcal{L}$-sequent is a tuple $\langle\Gamma, \varphi\rangle$ consisting of an $\mathcal{L}$-theory $\Gamma$ and an $\mathcal{L}$-sentence $\varphi$. A model of an $\mathcal{L}$-theory 
$\Gamma$ is a model in which all members of $\Gamma$ are true.

To state the two-cardinal problem, fix a unary relation symbol $U$ of $\sigma$, and say that an $\mathcal{L}$-theory $\Gamma$ admits $\langle\kappa, \lambda\rangle$, for cardinals $\kappa$ and $\lambda$, if there is a model $\mathfrak{A}=\langle D, e\rangle$ of $\Gamma$ such that $|D|=\kappa$ and $|e(U)|=\lambda$. The two-cardinal problem is to determine under which conditions an $\mathcal{L}$-theory admitting a given pair of infinite cardinals admits another given pair of infinite cardinals. One might think of this as the problem of extending the standard Löwenheim-Skolem theorem to the case of two cardinals, one for the domain and one for the interpretation of $U$; this was in fact how Robert Vaught introduced the problem (Morley and Vaught, 1962, section 6). Let $T_{\kappa, \lambda}$ be the set of $\mathcal{L}$-theories admitting $\langle\kappa, \lambda\rangle$, and $T_{\kappa, \leq \lambda}=\bigcup_{\aleph_{0} \leq \lambda^{\prime} \leq \lambda} T_{\kappa, \lambda^{\prime}}$.

From now on, let $\lambda, \kappa, \lambda^{\prime}, \kappa^{\prime}$, etc. always be infinite cardinals. The following two elementary facts for any $\mathcal{L}$-theory $\Gamma$ will be required:

Fact (i). If $\Gamma$ admits $\langle\kappa, \lambda\rangle$ and $\lambda \leq \kappa^{\prime} \leq \kappa$, then $\Gamma$ admits $\left\langle\kappa^{\prime}, \lambda\right\rangle$.

Fact (ii). If $\Gamma$ admits $\langle\kappa, \lambda\rangle$ then $\Gamma$ admits $\left\langle\kappa^{\prime}, \kappa^{\prime}\right\rangle$.

They can be derived from the Löwenheim-Skolem theorem for first-order logic; proofs can be found in Chang and Keisler (1990, Proposition 3.2.7 (i) and (ii)).

Membership in $\Lambda_{\kappa, \lambda}$ and $\vDash_{\kappa, \lambda}$ is straightforwardly reduced to satisfiability on $\langle\kappa, \lambda\rangle$ :

Lemma 1. Let $\langle\Gamma, \varphi\rangle$ be an $\mathcal{L}_{\square \text {-sequent. }}$

(i) $\Gamma \vDash_{\kappa, \lambda} \varphi$ if and only if $\Gamma \cup\{\neg \varphi\}$ is not satisfiable on $\langle\kappa, \lambda\rangle$.

(i) $\varphi \in \Lambda_{\kappa, \lambda}$ if and only if $\neg \varphi$ is not satisfiable on $\langle\kappa, \lambda\rangle$.

Proof. Immediate.

This lemma makes the connection to extensions of the Löwenheim-Skolem theorem precise. It should be noted that the particular class of Kripke models used here matters. E.g., if an accessibility relation and variable individual domains were introduced, it would be straightforward to prove that the same $\mathcal{L}_{\square}$-theories can be satisfied on all pairs of infinite cardinals, since one could always add an unconnected world with an arbitrary number of new individuals. Of course, there are also more interesting questions one could pose in such a setting; see, e.g., Bowen (1979, chapter 5). Such variant classes of models won't be considered in the following. Similarly, it is also essential to the results established below exactly which resources are available in $\mathcal{L}_{\square}$. E.g., adding further modal operators, such as explicit quantifiers over worlds, would substantially change the dependence of $\Lambda_{\kappa, \lambda}$ and $\vDash_{\kappa, \lambda}$ on $\kappa$ and $\lambda$.

The main results to be established in this section are Theorems 3 and 6 . The first reduces an $\mathcal{L}_{\square}$-theory being satisfiable on $\langle\kappa, \lambda\rangle$ to a corresponding $\mathcal{L}$-theory being a member of $T_{\kappa+\lambda, \leq \lambda}$, and the second reduces an $\mathcal{L}$-theory being a member of $T_{\kappa+\lambda, \leq \lambda}$ to a corresponding $\mathcal{L}_{\square}$-theory being satisfiable on $\langle\kappa, \lambda\rangle$. With various two-cardinal results from model theory, these two theorems will entail many useful corollaries, which will be derived in the two subsequent sections. To prove Theorems 3 and 6, observe first that since in any Kripke model, adding any number of copies of one of its worlds doesn't change which $\mathcal{L}_{\square}$-sentences are true in it, increasing the number of worlds in a frame doesn't destroy satisfiability: 


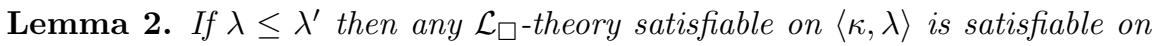
$\left\langle\kappa, \lambda^{\prime}\right\rangle$.

Proof. Assume $\lambda \leq \lambda^{\prime}$ and let $\Gamma$ be an $\mathcal{L}_{\square}$-theory satisfiable on $\langle\kappa, \lambda\rangle$. Let $\mathfrak{M}=\langle\kappa, \lambda, i$,@ $\rangle$ be a Kripke model of $\Gamma$. Define $\mathfrak{M}^{\prime}=\left\langle\kappa, \lambda^{\prime}, i^{\prime}, @\right\rangle$ such that for all relation symbols $R$ in $\sigma$ and $w<\lambda^{\prime}$,

$i^{\prime}(R)(w):= \begin{cases}i(R)(w) & \text { if } w<\lambda \\ i(R)(@) & \text { otherwise }\end{cases}$

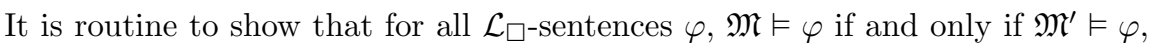
and so that $\mathfrak{M}^{\prime}$ is a Kripke model of $\Gamma$.

The syntactic mapping from $\mathcal{L}_{\square}$-theories to $\mathcal{L}$-theories used in Theorem 3 is a version of the so-called standard translation, which translates necessity as explicit universal quantification (informally, over worlds). To do so, the arity of each relation symbol is increased by one, and each atomic predication is translated by adding a special variable $\vartheta$ to the arguments (which can informally be understood as recording the world of evaluation). Necessity is translated using a universal quantifier binding $\vartheta$, restricted to a new relation symbol $U$ (informally, the worlds). Quantifiers are translated by restricting them to the negation of $U$ (informally, the individuals). Using this recursive translation, which will be called $\rho$, the present version of the standard translation, called $\tau$, is derived in a second step using a new unary relation symbol $A$ (which informally can be understood as identifying the actual world): $\tau(\varphi)$ is the sentence which says that there is a unique $U$ which is $A$, and letting $\vartheta$ be this element, $\rho(\varphi)$ is true.

To define this procedure more precisely, fix a unary relation symbol $A$ of $\sigma$ which is distinct from $U$, and $\vartheta \in V$. Let $\rho$ be an injection which maps every $n$ ary relation symbol of $\sigma$ to an $n+1$-ary relation symbol of $\sigma$, and every element of $V$ to an element of $V$, such that none of $U, A$ and $\vartheta$ are in its image. Extend $\rho$ to a mapping from $\mathcal{L}_{\square}$-formulas to $\mathcal{L}$-formulas using the following recursion clauses:

$$
\begin{aligned}
& \rho\left(R x_{1} \ldots x_{n}\right):=\rho(R) \rho\left(x_{1}\right) \ldots \rho\left(x_{n}\right) \vartheta \\
& \rho(x=y):=\rho(x)=\rho(y) \\
& \rho(\neg \varphi):=\neg \rho(\varphi) \\
& \rho(\varphi \wedge \psi):=\rho(\varphi) \wedge \rho(\psi) \\
& \rho(\square \varphi):=\forall \vartheta(U \vartheta \rightarrow \rho(\varphi)) \\
& \rho(\forall x \varphi):=\forall \rho(x)(\neg U \rho(x) \rightarrow \rho(\varphi))
\end{aligned}
$$

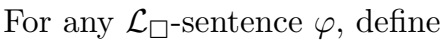

$\tau(\varphi):=\exists x(U x \wedge \forall y(A y \leftrightarrow x=y)) \wedge \forall \vartheta(A \vartheta \rightarrow \rho(\varphi))$

and extend $\tau$ in the obvious way to any $\mathcal{L}_{\square}$-theory $\Gamma$ :

$\tau(\Gamma):=\{\tau(\varphi): \varphi \in \Gamma\}$. 
To prove Theorem 3, two model transformations corresponding to the syntactic mapping $\tau$ will be used, transforming a Kripke model into a corresponding model, and vice versa:

Theorem 3. For any $\mathcal{L}_{\square}$-theory $\Gamma, \Gamma$ is satisfiable on $\langle\kappa, \lambda\rangle$ if and only if $\tau(\Gamma) \in T_{\kappa+\lambda, \leq \lambda}$.

Proof. Let $\Gamma \neq \emptyset$; otherwise, the claim is trivial. Assume first that $\Gamma$ is satisfiable on $\langle\kappa, \lambda\rangle$, witnessed by $\mathfrak{M}=\langle\kappa, \lambda, i, @\rangle$. Writing $X \uplus Y$ for the disjoint union of sets $X$ and $Y$, let $\mathfrak{A}=\langle\kappa \uplus \lambda, e\rangle$ be a model such that $e(U)=\lambda, e(A)=\{@\}$, and $e(\rho(R))=\left\{\left\langle o_{1}, \ldots, o_{n}, w\right\rangle:\left\langle o_{1}, \ldots, o_{n}\right\rangle \in i(R)(w), w<\lambda\right\}$ for any $n$ ary relation symbol $R$ in $\sigma$. A routine induction on the complexity of formulas

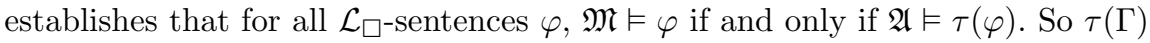
admits $\langle\kappa+\lambda, \lambda\rangle$ and thus is a member of $T_{\kappa+\lambda, \leq \lambda}$.

Assume now that $\tau(\Gamma) \in T_{\kappa+\lambda,<\lambda}$. Then for some $\lambda^{\prime} \leq \lambda, \tau(\Gamma)$ admits $\left\langle\kappa+\lambda, \lambda^{\prime}\right\rangle$. By Fact (i), $\tau(\Gamma)$ admits $\left\langle\kappa+\lambda^{\prime}, \lambda^{\prime}\right\rangle$. Let $\mathfrak{A}=\left\langle\kappa \uplus \lambda^{\prime}, e\right\rangle$ be a witness such that $e(U)=\lambda^{\prime}$. Let $\mathfrak{M}=\left\langle\kappa, \lambda^{\prime}, i\right.$, $\rangle$ be a Kripke model such that for every $n$-ary relation symbols $R$ in $\sigma$ and $w<\lambda^{\prime}, i(R)(w)=\left\{\left\langle o_{1}, \ldots, o_{n}\right\rangle\right.$ : $\left.\left\langle o_{1}, \ldots, o_{n}, w\right\rangle \in e(\rho(R))\right\}$, and @ is such that $e(A)=\{@\}$ (recall that $\Gamma$ is nonempty). Again, a routine induction on the complexity of formulas establishes

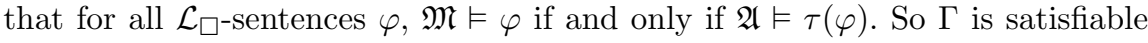
on $\left\langle\kappa, \lambda^{\prime}\right\rangle$, and thus by Lemma $2, \Gamma$ is satisfiable on $\langle\kappa, \lambda\rangle$.

For Theorem 6, a complementary mapping from $\mathcal{L}$-sentences to $\mathcal{L}_{\square}$-sentences will be used. The idea behind this translation is to add two constraints: on the one hand, for there to be at least as many worlds as $U \mathrm{~s}$, and on the other hand, for there to be infinitely many $U$ s. The first can be achieved by stating that each $U$ is possibly the unique $U$. To achieve the second, a new relation symbol $R$ will be used, stating that it is a serial, transitive and asymmetric on $U$.

More precisely, let $\pi$ be an injection which maps every $n$-ary relation symbol of $\sigma$ to an $n$-ary relation symbol of $\sigma$ distinct from $R$, and which maps $U$ to itself. Extend $\pi$ to a function from $\mathcal{L}$-formulas to $\mathcal{L}$-formulas which only replaces a relation symbol $F$ by $\pi(F)$. For any $\mathcal{L}$-sentence $\varphi$, define

$\mu(\varphi):=\pi(\varphi) \wedge \delta \wedge \iota$

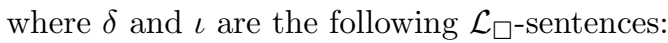

$$
\begin{aligned}
\delta:= & \forall x(U x \rightarrow \diamond \forall y(U y \leftrightarrow x=y)) \\
\iota:= & \forall x(U x \rightarrow \exists y(U y \wedge R x y)) \wedge \\
& \forall x \forall y \forall z((U x \wedge U y \wedge U z \wedge R x y \wedge R y z) \rightarrow R x z) \wedge \\
& \forall x \forall y((U x \wedge U y \wedge R x y) \rightarrow \neg R y x)
\end{aligned}
$$

As with $\tau$, extend $\mu$ in the obvious way to any $\mathcal{L}$-theory $\Gamma$ :

$\mu(\Gamma):=\{\mu(\varphi): \varphi \in \Gamma\}$.

To prove Theorem 6, two auxiliary lemmas are established:

Lemma 4. For any $\mathcal{L}$-theory $\Gamma$, if $\Gamma$ admits $\langle\kappa, \lambda\rangle$ then $\mu(\Gamma)$ is satisfiable on $\langle\kappa, \lambda\rangle$. 
Proof. Assume that $\Gamma$ admits $\langle\kappa, \lambda\rangle$, witnessed by a model $\mathfrak{A}=\langle\kappa, e\rangle$. Let $\mathfrak{M}=\langle\kappa, \lambda+1, i, \lambda\rangle$ (using ordinal addition) be a Kripke model such that for all $w<\lambda, i(U)(w)=\{\langle w\rangle\}, i(R)(\lambda)=\{\langle u, v\rangle: u<v<\lambda\}$, and, for any relation symbol $F$ in $\sigma, i(\pi(F))(\lambda)=e(F)$. It is routine to show that for any $\mathcal{L}$-sentence $\varphi, \mathfrak{A} \vDash \varphi$ if and only if $\mathfrak{M} \vDash \mu(\varphi)$, and so that $\mathfrak{M}$ is a Kripke model of $\mu(\Gamma)$. Since $|\lambda+1|=\lambda, \mu(\Gamma)$ is satisfiable on $\langle\kappa, \lambda\rangle$.

Lemma 5. For any $\mathcal{L}$-theory $\Gamma$, if $\mu(\Gamma)$ is satisfiable on $\langle\kappa, \lambda\rangle$ then $\Gamma$ admits $\left\langle\kappa, \lambda^{\prime}\right\rangle$ for some $\lambda^{\prime} \leq \lambda$.

Proof. Let $\Gamma \neq \emptyset$; otherwise, the claim is trivial. Let $\mathfrak{M}=\langle\kappa, \lambda, i$, @ $\rangle$ be a Kripke model witnessing that $\mu(\Gamma)$ is satisfiable on $\langle\kappa, \lambda\rangle$. Define a model $\mathfrak{A}=\langle\kappa, e\rangle$ by letting $e(F)=i(\pi(F))(@)$ for any relation symbol $F$ in $\sigma \cdot \mathfrak{A}$ is a model of $\Gamma$. Furthermore, by $\iota, \aleph_{0} \leq|e(U)|$, and by $\delta,|e(U)| \leq \lambda$ (recall that $\Gamma \neq \emptyset$ ).

Theorem 6. For any $\mathcal{L}$-theory $\Gamma, \Gamma \in T_{\kappa+\lambda, \leq \lambda}$ if and only if $\mu(\Gamma)$ is satisfiable on $\langle\kappa, \lambda\rangle$.

Proof. Assume first that $\Gamma \in T_{\kappa+\lambda, \leq \lambda}$. Then there is a $\lambda^{\prime} \leq \lambda$ such that $\Gamma$ admits $\left\langle\kappa+\lambda, \lambda^{\prime}\right\rangle$. If $\lambda>\kappa$, then note that by $\operatorname{Fact}(\mathrm{ii}), \Gamma$ admits $\langle\kappa, \kappa\rangle$, and so by Lemma $4, \mu(\Gamma)$ is satisfiable on $\langle\kappa, \kappa\rangle$, and so by Lemma $2, \mu(\Gamma)$ is satisfiable on $\langle\kappa, \lambda\rangle$. If $\lambda \leq \kappa$, then $\kappa+\lambda=\kappa$, so $\Gamma$ admits $\left\langle\kappa, \lambda^{\prime}\right\rangle$. So by Lemma $4, \mu(\Gamma)$ is satisfiable on $\left\langle\kappa, \lambda^{\prime}\right\rangle$ and thus by Lemma $2, \mu(\Gamma)$ is satisfiable on $\langle\kappa, \lambda\rangle$.

Assume now that $\mu(\Gamma)$ is satisfiable on $\langle\kappa, \lambda\rangle$. Then by Lemma $5, \Gamma$ admits $\left\langle\kappa, \lambda^{\prime}\right\rangle$ for some $\lambda^{\prime} \leq \lambda$. If $\lambda>\kappa$, then note that by Fact (ii), $\Gamma$ admits $\langle\lambda, \lambda\rangle$, and since then $\kappa+\lambda=\lambda$, $\Gamma$ admits $\langle\kappa+\lambda, \lambda\rangle$ and thus $\Gamma \in T_{\kappa+\lambda, \leq \lambda}$. If $\lambda \leq \kappa$, then $\kappa+\lambda=\kappa$, so $\Gamma$ admits $\left\langle\kappa+\lambda, \lambda^{\prime}\right\rangle$, and thus $\Gamma \in T_{\kappa+\lambda, \leq \lambda}$.

\subsection{Logics and Consequence Relations}

A helpful way of mapping out the logics and consequence relations determined by pairs of infinite cardinals, which also lends itself to reformulations in terms of analogs of the Löwenheim-Skolem theorem, is to establish conditions under which one is contained in another. Using Theorems 3 and 6, this can be reduced to the two-cardinal problem:

Theorem 7. (i) $\vDash_{\kappa^{\prime}, \lambda^{\prime}} \subseteq \vDash_{\kappa, \lambda}$ if and only if $T_{\kappa+\lambda, \leq \lambda} \subseteq T_{\kappa^{\prime}+\lambda^{\prime}, \leq \lambda^{\prime}}$.

(ii) $\Lambda_{\kappa^{\prime}, \lambda^{\prime}} \subseteq \Lambda_{\kappa, \lambda}$ if and only if for every $\mathcal{L}$-sentence $\varphi$, if $\{\varphi\} \in T_{\kappa+\lambda, \leq \lambda}$ then $\{\varphi\} \in T_{\kappa^{\prime}+\lambda^{\prime}, \leq \lambda^{\prime}}$

Proof. (i): If $\vDash_{\kappa^{\prime}, \lambda^{\prime}} \nsubseteq \vDash_{\kappa, \lambda}$, then by Lemma 1 , there is an $\mathcal{L}_{\square}$-theory $\Gamma$ which is satisfiable on $\langle\kappa, \lambda\rangle$ but not satisfiable on $\left\langle\kappa^{\prime}, \lambda^{\prime}\right\rangle$. By Theorem $3, \tau(\Gamma) \in T_{\kappa+\lambda, \leq \lambda}$ and $\tau(\Gamma) \notin T_{\kappa^{\prime}+\lambda^{\prime}, \leq \lambda^{\prime}}$, so $T_{\kappa+\lambda, \leq \lambda} \nsubseteq T_{\kappa^{\prime}+\lambda^{\prime}, \leq \lambda^{\prime}}$.

If $T_{\kappa+\lambda, \leq \lambda} \nsubseteq T_{\kappa^{\prime}+\lambda^{\prime}, \leq \lambda^{\prime}}$, then there is an $\mathcal{L}$-theory $\Gamma$ such that $\Gamma \in T_{\kappa+\lambda, \leq \lambda}$ and $\Gamma \notin T_{\kappa^{\prime}+\lambda^{\prime}, \leq \lambda^{\prime}}$. By Theorem $6, \mu(\Gamma)$ is satisfiable on $\langle\kappa, \lambda\rangle$ but not satisfiable on $\left\langle\kappa^{\prime}, \lambda^{\prime}\right\rangle$, so by Lemma $1, \vDash_{\kappa^{\prime}, \lambda^{\prime}} \nsubseteq \vDash_{\kappa, \lambda}$.

(ii): Analogous.

As a first corollary, it follows immediately that a frame in which there are at least as many worlds as individuals determines the unique weakest logic and consequence relation determined by any pair of infinite cardinals: 
Corollary 8. If $\kappa \leq \lambda$ then $\vDash_{\kappa, \lambda} \subseteq \vDash_{\kappa^{\prime}, \lambda^{\prime}}$ (and so $\Lambda_{\kappa, \lambda} \subseteq \Lambda_{\kappa^{\prime}, \lambda^{\prime}}$ ).

Proof. Assume $\kappa \leq \lambda$. Fact (ii) entails that for any $\mathcal{L}$-theory $\Gamma$, if $\Gamma \in T_{\kappa^{\prime}+\lambda^{\prime}, \leq \lambda^{\prime}}$ then $\Gamma$ admits $\langle\lambda, \lambda\rangle$, and therefore $\Gamma \in T_{\kappa+\lambda, \leq \lambda}$. So $T_{\kappa^{\prime}+\lambda^{\prime}, \leq \lambda^{\prime}} \subseteq T_{\kappa+\lambda, \leq \lambda}$ and therefore, with Theorem $7, \vDash_{\kappa, \lambda} \subseteq \vDash_{\kappa^{\prime}, \lambda^{\prime}}$.

A second corollary follows from the so-called infinite gap two-cardinal theorem, due to Vaught (1965a) (see also Chang and Keisler (1990, Theorem 7.2.6)), which entails that if a theory admits $\langle\kappa, \lambda\rangle$ which are sufficiently far apart, it admits $\left\langle\kappa^{\prime}, \lambda^{\prime}\right\rangle$. For this and the next few corollaries, two precise notions of distance among cardinals are required; one is in terms of successor cardinals, and one in terms of the powerset operation. So define, for any ordinal $\alpha, \aleph_{\alpha}(\kappa)$ and $\beth_{\alpha}(\kappa)$ as follows: $\aleph_{0}(\kappa)=\beth_{0}(\kappa)=\kappa ; \aleph_{\alpha+1}(\kappa)=\aleph_{\alpha}(\kappa)^{+}$and $\beth_{\alpha+1}(\kappa)=2^{\beth_{\alpha}(\kappa)}$; and, if $\alpha$ is a limit ordinal, $\aleph_{\alpha}(\kappa)=\bigcup_{\beta<\alpha} \aleph_{\beta}(\kappa)$ and $\beth_{\alpha}(\kappa)=\bigcup_{\beta<\alpha} \beth_{\beta}(\kappa)$. The relevant consequence of the infinite gap two-cardinal theorem can now formulated as saying that an $\mathcal{L}$-theory admitting $\left\langle\beth_{\omega}\left(\lambda^{\prime}\right), \lambda^{\prime}\right\rangle$ also admits $\langle\kappa, \lambda\rangle$ (for $\lambda \leq \kappa)$. It follows that any frame in which there are sufficiently more individuals than worlds determines the unique strongest logic and consequence relation determined by any pair of infinite cardinals:

Corollary 9. If $\kappa \geq \beth_{\omega}(\lambda)$, then $\vDash_{\kappa^{\prime}, \lambda^{\prime}} \subseteq \vDash_{\kappa, \lambda}$ (and so $\Lambda_{\kappa^{\prime}, \lambda^{\prime}} \subseteq \Lambda_{\kappa, \lambda}$ ).

Proof. Let $\kappa \geq \beth_{\omega}(\lambda)$, and consider any $\Gamma \in T_{\kappa+\lambda, \leq \lambda}$. Then there is a $\lambda^{*} \leq \lambda$ such that $\Gamma$ admits $\left\langle\kappa+\lambda, \lambda^{*}\right\rangle$. By Fact (i), $\Gamma$ admits $\left\langle\beth_{\omega}\left(\lambda^{*}\right), \lambda^{*}\right\rangle$ (as $\lambda^{*} \leq \lambda$, $\left.\lambda^{*} \leq \beth_{\omega}\left(\lambda^{*}\right) \leq \beth_{\omega}(\lambda) \leq \kappa+\lambda\right)$. By the infinite gap two-cardinal theorem, $\Gamma$ admits $\left\langle\kappa^{\prime}+\bar{\lambda}^{\prime}, \lambda^{\prime}\right\rangle$, and therefore $\Gamma \in T_{\kappa^{\prime}+\lambda^{\prime}, \leq \lambda^{\prime}}$. Hence $T_{\kappa+\lambda, \leq \lambda} \subseteq T_{\kappa^{\prime}+\lambda^{\prime}, \leq \lambda^{\prime}}$ and therefore by Theorem $7, \vDash_{\kappa^{\prime}, \lambda^{\prime}} \subseteq \vDash_{\kappa, \lambda}$.

An immediate consequence of these two corollaries is that if $\kappa \leq \lambda$, then $\vDash_{\kappa, \lambda}=\vDash_{\aleph_{0}, \aleph_{0}}$, and if $\kappa \geq \beth_{\omega}(\lambda)$, then $\vDash_{\kappa, \lambda}=\vDash_{\beth_{\omega}, \aleph_{0}}$, and analogously for the corresponding logics. To have non-arbitrary ways of referring to these, call them $\vDash_{\leq} / \Lambda_{\leq}$and $\vDash_{\gg} / \Lambda_{\gg}$, respectively.

What the first two corollaries leave open are $\vDash_{\kappa, \lambda}$ and $\Lambda_{\kappa, \lambda}$ where $\lambda<\kappa<$ $\beth_{\omega}(\lambda)$; call these "intermediate". The following two corollaries show that in this intermediate region, the comparative cardinalities matter; furthermore, in case the generalized continuum hypothesis (abbreviated GCH, the claim that $\aleph_{\alpha}=\beth_{\alpha}$ for all ordinals $\alpha$ ) fails, they show that both the comparison in terms of successor cardinals and the comparison in terms of the powerset operation matter.

Corollary 10. For each $n<\omega, \Lambda_{\beth_{n}(\lambda)^{+}, \lambda} \nsubseteq \Lambda_{\beth_{n}(\kappa), \kappa}$ (and so $\vDash_{\beth_{n}(\lambda)^{+}, \lambda} \nsubseteq$ $\left.\vDash_{\beth_{n}(\kappa), \kappa}\right)$.

Proof. There is an $\mathcal{L}$-sentence $\beta_{n}$ which admits, for every $\lambda^{\prime},\left\langle\beth_{n}\left(\lambda^{\prime}\right), \lambda^{\prime}\right\rangle$ but not $\left\langle\beth_{n}\left(\lambda^{\prime}\right)^{+}, \lambda^{\prime}\right\rangle$ (such a sentence is specified in the proof of Chang and Keisler (1990, Proposition 3.2.7 (iii), attributed to Raphael Robinson, unpublished)). So $\beta_{n}$ admits $\left\langle\beth_{n}(\kappa), \kappa\right\rangle$ and thus $\left\{\beta_{n}\right\} \in T_{\beth_{n}(\kappa), \leq \kappa}$. Assume for contradiction that $\left\{\beta_{n}\right\} \in T_{\beth_{n}(\lambda)^{+}, \leq \lambda}$. Then there is a $\lambda^{\prime} \leq \lambda$ such that $\beta_{n}$ admits $\left\langle\beth_{n}(\lambda)^{+}, \lambda^{\prime}\right\rangle$, and so by Fact (i), $\beta_{n}$ admits $\left\langle\beth_{n}\left(\lambda^{\prime}\right)^{+}, \lambda^{\prime}\right\rangle$ (since $\left.\lambda^{\prime} \leq \lambda, \lambda^{\prime} \leq \beth_{n}\left(\lambda^{\prime}\right)^{+} \leq \beth_{n}(\lambda)^{+}\right)$. 々, so $\left\{\beta_{n}\right\} \notin T_{\beth_{n}(\lambda)^{+}, \leq \lambda}$. Thus $\left\{\beta_{n}\right\} \in T_{\beth_{n}(\kappa), \leq \kappa}$ and $\left\{\beta_{n}\right\} \notin T_{\beth_{n}(\lambda)^{+}, \leq \lambda}$. With Theorem 6 , it follows that $\mu\left(\beta_{n}\right)$ is satisfiable on $\left\langle\beth_{n}(\kappa), \kappa\right\rangle$ but not $\left\langle\beth_{n}(\lambda)^{+}, \lambda\right\rangle$, and so that $\neg \mu\left(\beta_{n}\right)$ witnesses $\Lambda_{\beth_{n}(\lambda)^{+}, \lambda} \nsubseteq \Lambda_{\beth_{n}(\kappa), \kappa}$. 
While (Chang and Keisler, 1990, Proposition 3.2.7 (iv), attributed to Michael Morley, unpublished) record the existence of an $\mathcal{L}$-theory which admits, for every $\lambda,\left\langle\aleph_{n}(\lambda), \lambda\right\rangle$ but not $\left\langle\aleph_{n}(\lambda)^{+}, \lambda\right\rangle$, the construction of such a theory is left as an exercise to the reader. To ensure that the corresponding analog to Corollary 10 holds for logics, not just consequence relations, the following lemma shows that there is a finite such theory.

Lemma 11. For each $n<\omega$ there is an $\mathcal{L}$-sentence $\alpha_{n}$ which admits, for every $\lambda,\left\langle\aleph_{n}(\lambda), \lambda\right\rangle$ but not $\left\langle\aleph_{n}(\lambda)^{+}, \lambda\right\rangle$.

Proof. The following illustrates the proof for $n=1$; the general case is obtained by iterating the construction. Let $R$ be a binary and $B$ a ternary relation symbol of $\sigma$. Let $\varphi$ be the following $\mathcal{L}$-sentence, which requires $R$ to be a linear order and $B$ to induce, for every $x$, an injection from the elements $R$-related to $x$ to $U$ :

$$
\begin{aligned}
& \alpha_{1}:=\forall x \forall y((R x y \wedge R y x) \rightarrow x=y) \wedge \\
& \forall z((R x y \wedge R y z) \rightarrow R x z) \wedge \\
&\quad(R x y \vee R y x)) \wedge \\
& \forall x\left(\forall y\left(R y x \rightarrow \exists z\left(U z \wedge \forall z^{\prime}\left(B x y z^{\prime} \leftrightarrow z=z^{\prime}\right)\right)\right) \wedge\right. \\
&\left.\forall y \forall y^{\prime} \forall z\left(\left(B x y z \wedge B x y^{\prime} z\right) \rightarrow y=y^{\prime}\right)\right)
\end{aligned}
$$

$\alpha_{1}$ admits $\left\langle\lambda^{+}, \lambda\right\rangle$ : Interpret $R$ as the standard order on ordinals. For each $a<\lambda^{+}$, choose an injection $i_{a}: a \rightarrow \lambda$ (which exists since $|a| \leq \lambda$ ), and interpret $B$ to relate $a b c$ just in case $i_{a}(b)=c$.

$\alpha_{1}$ does not admit $\left\langle\lambda^{++}, \lambda\right\rangle$ : Assume $R$ is interpreted as a linear order, and let $a$ be an element which is above $\lambda^{+}$elements in this order (such an element must exist for cardinality reasons). Thus there can be no injection from these elements to $\lambda$, and so no interpretation verifying $\alpha_{1}$.

Corollary 12. For each $n<\omega, \Lambda_{\aleph_{n}(\lambda)^{+}, \lambda} \nsubseteq \Lambda_{\aleph_{n}(\kappa), \kappa}$ (and so $\vDash_{\aleph_{n}(\lambda)^{+}, \lambda} \nsubseteq$ $\left.\vDash_{\aleph_{n}(\kappa), \kappa}\right)$.

Proof. Analogous to the proof of Corollary 10, using Lemma 11.

In this proof, $\neg \mu\left(\alpha_{1}\right)$ witnesses $\Lambda_{\lambda^{++}, \lambda} \nsubseteq \Lambda_{\kappa^{+},{ }_{\kappa}}$. What does this sentence look like? Apart from relabeling $R$ in $\alpha_{1}, \mu$ conjoins $\alpha_{1}$ with two $\mathcal{L}_{\square \text {-sentences } \delta}$ and $\iota$ which can informally be understood as requiring there to be infinitely many $U$ s and there to be a world corresponding to each $U$. The most straightforward way of understanding how $\neg \mu\left(\alpha_{1}\right)$ imposes a constraint on the relation between cardinalities of individuals and worlds is in two steps, one imposing a constraint on the relation between cardinalities of individuals and $U$ s and one imposing a constraint on the relation between cardinalities of $U_{\mathrm{s}}$ and worlds. This line of thought applies as well to the witnesses used in the proof of Corollary 10 above and the proofs of several further corollaries below.

Especially without the GCH, the last two corollaries show that the intermediate region exhibits interesting structure. The following two corollaries impose two basic containment properties on the whole space, and so in particular on this intermediate region. The first simply applies the earlier observation that satisfiability can't be destroyed by increasing the number of worlds; the second notes that it can also not be destroyed by reducing the number of individuals: 
Corollary 13. If $\lambda^{\prime} \leq \lambda$ then $\vDash_{\kappa, \lambda} \subseteq \vDash_{\kappa, \lambda^{\prime}}$ (and so $\Lambda_{\kappa, \lambda} \subseteq \Lambda_{\kappa, \lambda^{\prime}}$ ).

Proof. By Lemmas 1 and 2.

Corollary 14. If $\kappa^{\prime} \leq \kappa$ then $\vDash_{\kappa^{\prime}, \lambda} \subseteq \vDash_{\kappa, \lambda}$ (and so $\Lambda_{\kappa^{\prime}, \lambda} \subseteq \Lambda_{\kappa, \lambda}$ ).

Proof. Let $\kappa^{\prime} \leq \kappa$. Then $\kappa^{\prime}+\lambda \leq \kappa+\lambda$, so, by Fact (i), for all $\lambda^{\prime} \leq \lambda, T_{\kappa+\lambda, \lambda^{\prime}} \subseteq$ $T_{\kappa^{\prime}+\lambda, \lambda^{\prime}}$. So $T_{\kappa+\lambda, \leq \lambda} \subseteq T_{\kappa^{\prime}+\lambda, \leq \lambda}$, and therefore, by Theorem $7, \vDash_{\kappa^{\prime}, \lambda} \subseteq \vDash_{\kappa, \lambda}$.

The following diagram summarizes the positive corollaries obtained so far for logics and consequence relations determined by a pair of cardinals in the ב-series:

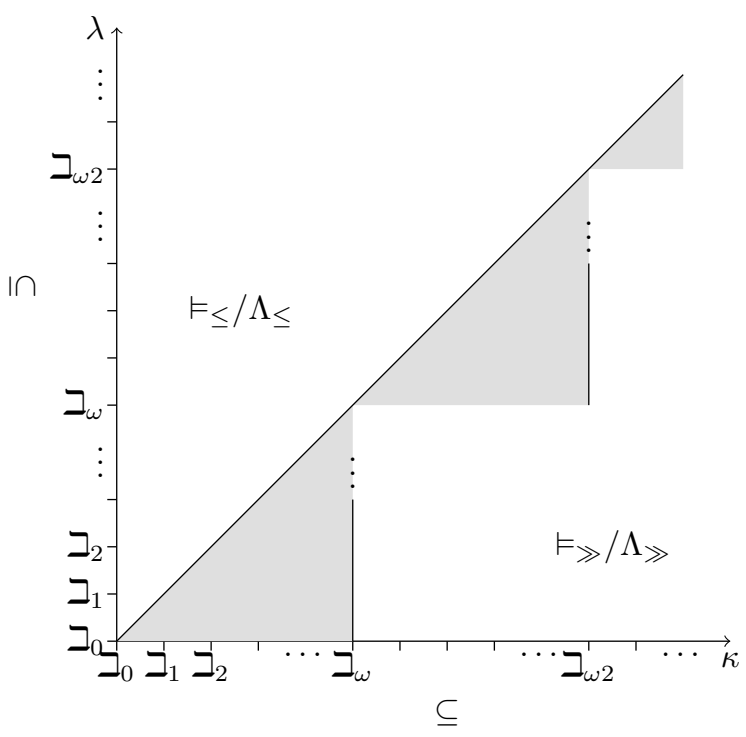

The two curves in the diagram divide the space of logics and consequence relations it represents into the region of $\vDash_{\leq}$, which includes those on diagonal

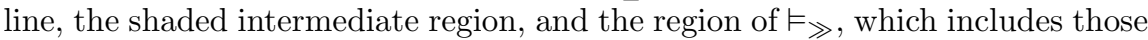
on the vertical lines. $\subseteq$ marks the containment properties along both axes established in the last two corollaries. It should be noted that the diagram might be misleading in two respect: First, it only records pairs of cardinals in the $\beth$-series, so unless the GCH is true, it does not represent all pairs of infinite cardinals. Second, although the shaded area and lines suggest continuity, the represented space is obviously discrete. Not represented in the diagram is Corollary 10, which entails that any two represented consequence relations in the intermediate region are distinct if they, as one might say, differ in how far they are from the diagonal.

Assuming the GCH, every intermediate consequence relations is identical to $\vDash_{\aleph_{n}(\kappa), \kappa}$ for some $\kappa$ and $n<\omega$. One might conjecture that in this case, at least the choice of $\kappa$ does not matter. But it turns out that this conjecture is unlikely to be settled by ZFC, even assuming the GCH: On the one hand, the conjecture follows from $V=L$, the axiom of constructibility, which entails the GCH. On the other hand, given the consistency of a very mild large cardinal hypothesis, $\mathrm{ZFC}+\mathrm{GCH}$ is consistent with the negation of the conjecture. 
For the first of these observation, the conjecture will be derived from the so-called gap $n$ conjecture, which says that every $\mathcal{L}$-theory admitting $\left\langle\aleph_{n}(\kappa), \kappa\right\rangle$ also admits $\left\langle\aleph_{n}(\lambda), \lambda\right\rangle$. According to Chang and Keisler (1990, p. 524), that this holds for all $n<\omega$ was shown to follow from $V=L$ in unpublished work by Ronald Jensen. (Details of the gap 1 case can be found in Jensen (1972); details of the gap 2 case and a sketch of the gap $n$ case can be found in Devlin (1984, chapter VIII).)

Corollary 15. If $V=L$, then for all $n<\omega, \vDash_{\aleph_{n}(\lambda), \lambda}=\vDash_{\aleph_{n}(\kappa), \kappa}$ (and so $\left.\Lambda_{\aleph_{n}(\lambda), \lambda}=\Lambda_{\aleph_{n}(\kappa), \kappa}\right)$.

Proof. Assume $V=L$ and $\Gamma \in T_{\aleph_{n}(\kappa), \leq \kappa}$. Then $\Gamma$ admits $\left\langle\aleph_{n}(\kappa), \kappa^{\prime}\right\rangle$ for some $\kappa^{\prime} \leq \kappa$, and thus $\left\langle\aleph_{n}\left(\kappa^{\prime}\right), \kappa^{\prime}\right\rangle$ by Fact (i). So by the gap $n$ conjecture, $\Gamma$ admits $\left\langle\aleph_{n}(\lambda), \lambda\right\rangle$ and therefore $\Gamma \in T_{\aleph_{n}(\lambda), \leq \lambda}$. So $T_{\aleph_{n}(\kappa), \leq \kappa} \subseteq T_{\aleph_{n}(\lambda), \leq \lambda}$, and thus, with Theorem $7, \vDash_{\aleph_{n}(\lambda), \lambda} \subseteq \vDash_{\aleph_{n}(\kappa), \kappa}$. By symmetry, $\vDash_{\aleph_{n}(\lambda), \lambda}=\vDash_{\aleph_{n}(\kappa), \kappa}$.

Assuming $V=L$, the logics and consequence relations therefore form $\omega+1$ chains, linearly ordered by the proper subset relation, and determined by the following sequence of pairs of cardinals:

$$
\left\langle\aleph_{0}, \aleph_{0}\right\rangle,\left\langle\aleph_{1}, \aleph_{0}\right\rangle,\left\langle\aleph_{2}, \aleph_{0}\right\rangle, \ldots,\left\langle\aleph_{\omega}, \aleph_{0}\right\rangle
$$

The second observation is based on a consistency proof of the failure of the gap 2 conjecture due to Jack Silver. This proof uses an $\mathcal{L}$-sentence $k$ which admits $\left\langle\kappa^{++}, \kappa\right\rangle$ if and only if a certain claim $K_{2}(\kappa)$ holds. $\left(K_{2}(\kappa)\right.$ states the existence of a so-called Kurepa tree satisfying certain properties in terms of $\kappa$. A sketch of the construction of $k$ can be found in Vaught (1965b); see also the discussion in Chang and Keisler (1990, p. 527).) Using $\mu$ and $\alpha_{2}$ (as in Lemma 11), this can be turned into a sentence which is a member of $\Lambda_{\kappa^{++},{ }_{\kappa}}$ if and only if $K_{2}(\kappa)$ fails:

Lemma 16. $\neg \mu\left(k \wedge \alpha_{2}\right) \in \Lambda_{\kappa^{++}, \kappa}$ if and only if $\neg K_{2}(\kappa)$.

Proof. $\neg \mu\left(k \wedge \alpha_{2}\right) \in \Lambda_{\kappa^{++}, \kappa}$ if and only if $\mu\left(k \wedge \alpha_{2}\right)$ is not satisfiable on $\left\langle\kappa^{++}, \kappa\right\rangle$. By Theorem 6 , this is the case if and only if $\left\{k, \alpha_{2}\right\} \notin T_{\kappa^{++}, \leq \kappa}$. Since $\alpha_{2}$ does not admit $\left\langle\kappa^{++}, \kappa^{\prime}\right\rangle$ for any $\kappa^{\prime}<\kappa$, this is equivalent to the the claim that $k$ does not admit $\left\langle\kappa^{++}, \kappa\right\rangle$, which, as noted, is the case if and only if $\neg K_{2}(\kappa)$.

On the assumption that ZFC is consistent with the existence of two uncountable inaccessible cardinals, Silver (1971) has proven that ZFC + GCH is consistent with $K_{2}\left(\aleph_{1}\right) \wedge \neg K_{2}\left(\aleph_{0}\right)$ as well as consistent with $K_{2}\left(\aleph_{0}\right) \wedge \neg K_{2}\left(\aleph_{1}\right)$. Thus on this assumption, ZFC + GCH neither settles whether $\neg \mu\left(k \wedge \alpha_{2}\right)$ is a member of $\Lambda_{\aleph_{2}, \aleph_{0}}$, nor whether it is a member of $\Lambda_{\aleph_{3}, \aleph_{1}}$, nor whether these logics are the same:

Corollary 17. If ZFC is consistent with the existence of two uncountable inaccessible cardinals, then $\mathrm{ZFC}+\mathrm{GCH}$ is consistent with (i) as well as consistent with (ii):

(i) $\Lambda_{\aleph_{2}, \aleph_{0}} \nsubseteq \Lambda_{\aleph_{3}, \aleph_{1}}$ (and so $\vDash_{\aleph_{2}, \aleph_{0}} \nsubseteq \vDash_{\aleph_{3}, \aleph_{1}}$ )

(ii) $\Lambda_{\aleph_{3}, \aleph_{1}} \nsubseteq \Lambda_{\aleph_{2}, \aleph_{0}}$ (and so $\vDash_{\aleph_{3}, \aleph_{1}} \nsubseteq \vDash_{\aleph_{2}, \aleph_{0}}$ )

Proof. Immediate by Lemma 16 and the result mentioned above. 
In the same way, an analogous negative result for $\left\langle\aleph_{3}, \aleph_{1}\right\rangle$ and $\left\langle\aleph_{7}, \aleph_{5}\right\rangle$ can be derived, and the independence of $\neg \mu\left(k \wedge \alpha_{2}\right) \in \Lambda_{\aleph_{2}, \aleph_{0}}$ from ZFC (rather than $\mathrm{ZFC}+\mathrm{GCH}$ ) can be obtained from the slightly weaker assumption that ZFC is consistent with the existence of one uncountable inaccessible cardinal. (See Chang and Keisler (1990, pp. 527-528), who attribute the relevant results to Silver and Robert Solovay.)

Some particular positive results have been obtained in the case of the gap 1 conjecture, which lead to some particular positive corollaries for logics and consequence relations determined by pairs of infinite cardinals $\left\langle\kappa^{+}, \kappa\right\rangle$, although negative results are also available for such pairs. The first positive corollary is based on what is known as Vaught's Two-Cardinal Theorem:

Corollary 18. If $\kappa>\lambda$, then $\vDash_{\aleph_{1}, \aleph_{0}} \subseteq \vDash_{\kappa, \lambda}$ (and so $\Lambda_{\aleph_{1}, \aleph_{0}} \subseteq \Lambda_{\kappa, \lambda}$ ).

Proof. As proven in Morley and Vaught (1962, Theorem 6.2) (see also Chang and Keisler (1990, Theorem 3.2.9)), for all $\lambda^{\prime} \leq \lambda, T_{\kappa, \lambda^{\prime}} \subseteq T_{\aleph_{1}, \aleph_{0}}$, so $T_{\kappa+\lambda, \leq \lambda} \subseteq$ $T_{\aleph_{1}, \aleph_{0}}$, and thus by Theorem $7, \vDash_{\aleph_{1}, \aleph_{0}} \subseteq \vDash_{\kappa, \lambda}$.

Using what is known as Chang's Two-Cardinal Theorem, this first positive corollary leads to a second one:

Corollary 19. If the GCH holds and $\kappa$ is regular, then $\vDash_{\kappa^{+},,_{\kappa}}=\vDash_{\aleph_{1}, \aleph_{0}}$ (and so $\left.\Lambda_{\kappa^{+}, \kappa}=\Lambda_{\aleph_{1}, \aleph_{0}}\right)$.

Proof. As proven in Chang (1965) (see also Chang and Keisler (1990, Theorem 7.2.7)), $T_{\aleph_{1}, \aleph_{0}} \subseteq T_{\kappa^{+}, \kappa}$, so $T_{\aleph_{1}, \aleph_{0}} \subseteq T_{\kappa^{+}, \leq \kappa}$, and thus by Theorem 7 , $\vDash_{\kappa^{+}, \kappa} \subseteq \vDash_{\aleph_{1}, \aleph_{0}}$. With Corollary 18, the claim follows.

The relevant negative results depend on large cardinal assumptions which are stronger than those used in the gap 2 case. Similar to the use of Kurepa trees in the gap 2 case, they use so-called special $\kappa^{+}$-Aronszajn trees, whose existence will be written as $A\left(\kappa^{+}\right)$. Again, there is an $\mathcal{L}$-sentence $a$ which admits $\left\langle\kappa^{+}, \kappa\right\rangle$ if and only if $A\left(\kappa^{+}\right)$(a construction is given in Chang and Keisler (1990, 7.2.11); the observation that such a sentence exists is attributed to Frederick Rowbottom and Silver, independently and unpublished).

Lemma 20. $\neg \mu\left(a \wedge \alpha_{1}\right) \in \Lambda_{\kappa^{+}, \kappa}$ if and only if $\neg A\left(\kappa^{+}\right)$.

Proof. Analogous to Lemma 16.

While $A\left(\aleph_{1}\right)$ holds, the consistency of ZFC with the existence of a Mahlo cardinal entails the consistency of ZFC with $\neg A\left(\aleph_{2}\right)$, and the consistency of ZFC with the existence of a supercompact cardinal entails the consistency of ZFC + GCH with $\neg A\left(\aleph_{\omega+1}\right)$ (see Chang and Keisler (1990, 7.2.10. (i), 7.2.12. (i) \& (iii)), who attribute these results to Nachman Aronszajn, Mitchell (1972) and Shelah (1979)). Thus:

Corollary 21. (i) If ZFC is consistent with the existence of a Mahlo cardinal, then ZFC is consistent with $\Lambda_{\aleph_{2}, \aleph_{1}} \nsubseteq \Lambda_{\aleph_{1}, \aleph_{0}}$ (and so $\vDash_{\aleph_{2}, \aleph_{1}} \nsubseteq \vDash_{\aleph_{1}, \aleph_{0}}$ ).

(ii) If ZFC is consistent with the existence of a supercompact cardinal, then $\mathrm{ZFC}+\mathrm{GCH}$ is consistent with $\Lambda_{\aleph_{\omega+1}, \aleph_{\omega}} \nsubseteq \Lambda_{\aleph_{1}, \aleph_{0}}$ (and so $\vDash_{\aleph_{\omega+1}, \aleph_{\omega}} \nsubseteq$ $\vDash_{\aleph_{1}, \aleph_{0}}$ ).

Proof. Immediate by Lemma 20 and the results mentioned above. 


\subsection{Axiomatizations}

If it could be argued that $\models_{\mathrm{MU}}$ is given by a particular pair $\langle\kappa, \lambda\rangle$, it would be interesting to specify it using a standard axiomatic calculus. This requires (i) $\vDash_{\kappa, \lambda}$ to be compact, (ii) $\vDash_{\kappa, \lambda}$ to satisfy the deduction/detachment property, and (iii) $\Lambda_{\kappa, \lambda}$ to be recursively enumerable. (ii) is easily seen to hold in general:

Proposition 22. $\Gamma \cup\{\varphi\} \vDash_{\kappa, \lambda} \psi$ if and only if $\Gamma \vDash_{\kappa, \lambda} \varphi \rightarrow \psi$.

Proof. Routine.

This raises the question which pairs of infinite cardinals determine compact consequence relations, and which determine recursively enumerable logics. For those pairs that satisfy both conditions, it is also interesting to consider whether there are any natural axiomatizations of the logics they determine. The next result shows that all of this is easily done in the case of $\vDash_{\leq}$. For concreteness, the construction will be based on the axiomatization described in Hughes and Cresswell (1996, chapter 13), which will be called LPC + S5. Its theorems are the same as those of the system which was presented in a more compressed manner in Kripke (1959), and there proven to be sound and complete with respect to the class of all frames.

Theorem 23. Let $\mathrm{LPC}^{\infty}+\mathrm{S} 5$ be the result of adding the following axiom schema to $\mathrm{LPC}+\mathrm{S} 5$ :

$E_{n}:=\exists x_{0} \ldots \exists x_{n-1} \bigwedge_{i<j<n} x_{i} \neq x_{j}$

Let $\vdash$ be the set of sequents $\langle\Gamma, \varphi\rangle$ such that $\bigwedge_{\gamma \in \Gamma_{0}} \gamma \rightarrow \varphi$ is derivable in $\mathrm{LPC}^{\infty}+\mathrm{S} 5$ for some finite $\Gamma_{0} \subseteq \Gamma . \vdash=\vDash_{\leq}$.

Proof. If $\Gamma \nvdash \varphi$, then $\Gamma \cup\left\{E_{n}: n<\omega\right\} \nvdash \varphi$, so there is no finite $\Delta \subseteq \Gamma \cup\left\{E_{n}\right.$ : $n<\omega\}$ such that $\bigwedge_{\delta \in \Delta} \delta \rightarrow \varphi$ is derivable in $\mathrm{LPC}^{\infty}+\mathrm{S} 5$, and so no such sentence is derivable in LPC $+\mathrm{S} 5$ either. Thus by the adequacy of LPC + S5 (Hughes and Cresswell, 1996, chapter 14), $\Gamma \cup\left\{E_{n}: n<\omega\right\} \cup\{\neg \varphi\}$ is satisfiable on a frame, which must contain infinitely many individuals. By Corollaries 13 and 8 , this can be assumed to be $\left\langle\aleph_{0}, \aleph_{0}\right\rangle$, so $\Gamma \not \nvdash_{\leq} \varphi$.

Since the axioms of $\mathrm{LPC}+\mathrm{S} 5$ are valid on all frames, and the instances of $E_{n}$ are valid on $\left\langle\aleph_{0}, \aleph_{0}\right\rangle$, the axioms of $\mathrm{LPC}^{\infty}+\mathrm{S} 5$ are valid on $\left\langle\aleph_{0}, \aleph_{0}\right\rangle$. The validities on $\left\langle\aleph_{0}, \aleph_{0}\right\rangle$ are easily seen to be closed under the rules of LPC + S5 (necessitation, modus ponens, and a version of universal generalization). Thus all sentences provable in $\mathrm{LPC}^{\infty}+\mathrm{S} 5$ are valid on $\left\langle\aleph_{0}, \aleph_{0}\right\rangle$. Now, if $\Gamma \vdash \varphi$, then $\bigwedge_{\gamma \in \Gamma_{0}} \gamma \rightarrow \varphi$ is derivable in $\mathrm{LPC}^{\infty}+\mathrm{S} 5$ for some finite $\Gamma_{0} \subseteq \Gamma$. Consider any model of $\Gamma$ based on $\left\langle\aleph_{0}, \aleph_{0}\right\rangle$; this must be a model of $\Gamma_{0}$, and so a model of $\varphi$, hence $\Gamma \vDash \leq \varphi$.

In case $\kappa>\lambda$, the issues are more subtle. Theorems 3 and 6 allow us to derive a number of results on compactness and recursive enumerability. Starting with compactness, they yield the following theorem, which reduces the compactness of $\vDash_{\kappa, \lambda}$ to a corresponding property for $T_{\kappa+\lambda, \leq \lambda}$ :

Theorem 24. The following are equivalent:

(i) $\vDash_{\kappa, \lambda}$ is compact. 
(ii) If every finite subset of an $\mathcal{L}$-theory $\Gamma$ is a member of $T_{\kappa+\lambda, \leq \lambda}$ then $\Gamma \in$ $T_{\kappa+\lambda, \leq \lambda}$.

Proof. Assume first that (ii) is not the case, witnessed by an $\mathcal{L}$-theory $\Gamma$. Then by Theorem $6, \mu(\Gamma)$ is not satisfiable on $\langle\kappa, \lambda\rangle$ although all of its finite subsets are; thus $\models_{\kappa, \lambda}$ is not compact.

Assume now that $\vDash_{\kappa, \lambda}$ is not compact, so there is an $\mathcal{L}_{\square}$-theory $\Gamma$ which is not satisfiable on $\langle\kappa, \lambda\rangle$ although all of its finite subsets are. By Theorem 3 , all finite subsets of $\tau(\Gamma)$ are members of $T_{\kappa+\lambda, \leq \lambda}$ but $\tau(\Gamma) \notin T_{\kappa+\lambda, \leq \lambda}$; thus (ii) is not the case.

For $\vDash_{\gg}$ and $\vDash_{\aleph_{1}, \aleph_{0}}$, results are available from which compactness follows:

Corollary 25. $\vDash_{\gg}$ and $\vDash_{\aleph_{1}, \aleph_{0}}$ are compact.

Proof. As shown in Vaught (1965a), if every finite subset of an $\mathcal{L}$-theory $\Gamma$ admits $\left\langle\beth_{\omega}, \aleph_{0}\right\rangle$ then $\Gamma$ admits $\left\langle\beth_{\omega}, \aleph_{0}\right\rangle$; see also Chang and Keisler (1990, Theorem 7.2.6, Exercise 7.2.12). As shown in Morley and Vaught (1962), if every finite subset of an $\mathcal{L}$-theory $\Gamma$ admits $\left\langle\aleph_{1}, \aleph_{0}\right\rangle$ then $\Gamma$ admits $\left\langle\aleph_{1}, \aleph_{0}\right\rangle$; see also Chang and Keisler (1990, Exercise 3.2.13). The claim follows by Theorem 24.

No corresponding compactness results seem easily available for the remaining intermediate consequence relations. Indeed, Shelah (2005) contains, for each $n>1$, a consistency result for the existence of an $\mathcal{L}$-theory which does not admit $\left\langle\aleph_{n}, \aleph_{0}\right\rangle$ although all of its finite subsets admit it. With Theorem 24, a corresponding consistency result for the incompactness of $\models_{\aleph_{n}, \aleph_{0}}$, for $n>1$, follows.

The question of recursive enumerability can also be reduced to one which has been explored in model theory, using the standard notion of consistency in first-order non-modal logic:

Theorem 26. If there is a recursive $\mathcal{L}$-theory $\Gamma$ such that for any $\mathcal{L}$-sentence $\varphi, \Gamma \cup\{\varphi\}$ is consistent if and only if $\{\varphi\} \in T_{\kappa+\lambda, \leq \lambda}$, then $\Lambda_{\kappa, \lambda}$ is recursively enumerable.

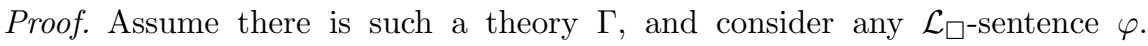
$\varphi \in \Lambda_{\kappa, \lambda}$ iff $\neg \varphi$ is not satisfiable on $\langle\kappa, \lambda\rangle$. By Theorem 3 , this is the case iff $\{\tau(\neg \varphi)\} \notin T_{\kappa+\lambda, \leq \lambda}$. By assumption, this is the case iff $\Gamma \cup\{\tau(\neg \varphi)\}$ is inconsistent, which is the case iff $\tau(\varphi)$ is derivable from $\Gamma$ in a standard calculus for first-order logic. This yields a method for recursively enumerating $\Lambda_{\kappa, \lambda}$.

Corollary 27. $\Lambda_{\gg}$ and $\Lambda_{\aleph_{1}, \aleph_{0}}$ are recursively enumerable.

Proof. For $\vDash_{\gg}$, let $\kappa=\beth_{\omega}$ and $\lambda=\aleph_{0}$. The existence of a set satisfying the condition on $\Gamma$ in Theorem 26 follows from the corresponding two-cardinal theorem by Vaught (1965a); explicit specifications of such sets are given in Barwise (1977) and Schmerl (1977). In the case of $\models_{\aleph_{1}, \aleph_{0}}$, the existence of a set satisfying the condition on $\Gamma$ in Theorem 26 for $\kappa=\aleph_{1}$ and $\lambda=\aleph_{0}$ was noted in Vaught (1964) based on results in Fuhrken (1964); an explicit specification of such a set is given in Keisler (1966). In each case, the recursive enumerability of the relevant set of theorems follows by Theorem 26 . 
As the preceding corollaries indicate, $\models_{\gg}$ and $\models_{\aleph_{1}, \aleph_{0}}$ stand out as compact consequence relations whose corresponding logics are easily shown to be recursively enumerable. It would be interesting to develop axiomatizations for them as well. Indeed, these two stand out also in that any of the other logics and consequence relations are likely to be extremely difficult to axiomatize; recall that previous corollaries exhibited various potential failures of ZFC (or even ZFC + GCH) to decide membership in logics such as $\Lambda_{\aleph_{2}, \aleph_{0}}, \Lambda_{\aleph_{2}, \aleph_{1}}$ and $\Lambda_{\aleph_{\omega+1}, \aleph_{\omega}}$.

LPC $^{\infty}+$ S5 is an infinitary axiomatization of $\Lambda_{\leq}$in the sense that it is obtained from LPC + S5 by adding an axiom schema with infinitely many instances. It turns out that this is an essential feature, in the sense that there is no finite set of axioms which added to LPC $+\mathrm{S} 5$ yields an axiomatization of $\Lambda_{\leq}$(even if a rule of substitution is added to LPC + S5, which Hughes and Cresswell (1996, chapter 13) do not include). Moreover, this is the case for any $\operatorname{logic} \Lambda_{\kappa, \lambda}$; in this sense, none of the logics under consideration here is finitely axiomatizable.

Without setting up the relevant variant of LPC + S5 in detail, here is a sketch of the proof: Consider a model $\mathfrak{M}$ based on $\langle\kappa, \lambda\rangle$ which interprets every $n$-ary relation symbol in $\sigma$ as the function mapping every element of $\lambda$ to $\kappa^{n}$, the set of all $n$-tuples on $\kappa$. For any $i<\omega$, define $\mathfrak{M}_{i}$ to be such a model based on $\langle i, \lambda\rangle$, i.e., one which interprets every $n$-ary relation symbol in $\sigma$ as the function mapping every element of $\lambda$ to $i^{n}$. Using a back and forth system, it can be shown that any $\mathcal{L}_{\square}$-sentence of quantifier rank up to $i$ is true in $\mathfrak{M}$ if and only if it is true in $\mathfrak{M}_{i}$. (The relevant definitions can be adapted from Fritz (2013).) Considering any finite set $\Gamma \subseteq \Lambda_{\kappa, \lambda}$, let $i$ be the maximum of the quantifier ranks of its members. Since $\mathfrak{M}$ verifies all members of $\Gamma$, so does $\mathfrak{M}_{i}$. All axioms LPC $+\mathrm{S} 5$ are true in $\mathfrak{M}_{i}$ (under any assignment function),

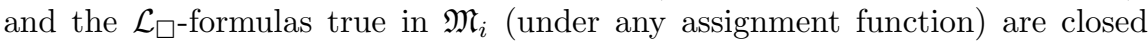
under the rules of LPC $+\mathrm{S} 5$. But $E_{i+1}$, a sentence saying that there are at least $i+1$ individuals, is not true in $\mathfrak{M}_{i}$. Thus $E_{i+1}$, which is a member of $\Lambda_{\kappa, \lambda}$, is not derivable in the axiomatization resulting from adding the members of $\Gamma$ to $\mathrm{LPC}+\mathrm{S} 5$.

\section{Metaphysical Universality}

Recall that the previous section developed the theory of logics and consequence relations determined by pairs of infinite cardinals on the assumption of firstorder ZFC, a theory of pure sets. Since there are non-sets, there is no pure set containing all individuals, and so no intended frame, i.e., no frame $\langle D, W\rangle$ such that $D$ is the set of individuals and $W$ is the set of worlds. (Even in a variant model theory based on one of the usual extensions of ZFC to impure sets, there is no intended frame, since there is no universal set.) An argument is therefore needed to show that $\Lambda_{\mathrm{MU}}$ and $\vDash_{\mathrm{MU}}$ are determined by some pair of infinite cardinals. Williamson (2013, pp. 117-118, 145) tentatively suggests such an argument, conditional on Premise 1, stated below.

The next section summarizes this argument, albeit somewhat loosely. The argument relies on two premises, which will be discussed in subsequent sections. On the one hand, some difficulties in evaluating the second premise of the argument will be raised. On the other hand, the first premise relies on the 
assumption that worlds are individuals; it will be shown how to eliminate this assumption.

\subsection{Williamson's Argument}

Let $\mathcal{L}^{2}$ be the extension of $\mathcal{L}$ by an infinite set of relational variables for each arity, as well as quantifiers binding them; let $\mathcal{L}_{\square}^{2}$ be the corresponding extension of $\mathcal{L}_{\square}$. For any $\mathcal{L}_{\square}$-formula $\varphi$, let $\forall \varphi$ be the result of uniformly replacing each relation symbol in $\varphi$ by a distinct relational variable of appropriate arity, and prefixing the resulting formula by a string of universal quantifiers binding all of these variables; the result is an $\mathcal{L}_{\square}^{2}$-formula. Unless explicitly noted otherwise, first- and second-order quantifiers will be interpreted unrestrictedly, and $\square$ as

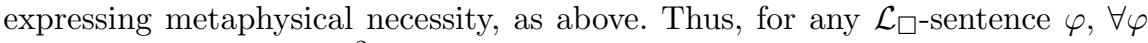
is a fully interpreted $\mathcal{L}_{\square}^{2}$-sentence. With this, the definition of $\Lambda_{\mathrm{MU}}$ can be summarized as follows:

$\Lambda_{\mathrm{MU}}=\left\{\varphi \in \mathcal{L}_{\square}: \forall \varphi\right.$ is true $\}$

Recall that this truth predicate is assumed to be transparent, as third- and in general higher-order quantifiers are assumed to be available in the metalanguage.

As in section 2.1 , let $\rho$ be an injection which maps every $n$-ary relation symbol of $\sigma$ to an $n+1$-ary relation symbol of $\sigma$, and every element of $V$ to an element of $V$, such that neither $U$ nor $\vartheta$ are in its image; this was extended to a version of the standard translation from $\mathcal{L}_{\square}$-formulas to $\mathcal{L}$-formulas. Instead of this extension of $\rho$, the following will use a variant extension $\zeta$ which differs from the above extension of $\rho$ only in that first-order quantifiers are not restricted to the negation of $U$. Thus, the most important clauses are:

$\zeta\left(R x_{1} \ldots x_{n}\right):=\rho(R) \rho\left(x_{1}\right) \ldots \rho\left(x_{n}\right) \vartheta$

$\zeta(\square \varphi):=\forall \vartheta(U \vartheta \rightarrow \zeta(\varphi))$

$\zeta(\forall x \varphi):=\forall \rho(x) \zeta(\varphi)$

Extend the notation $\forall \varphi$ introduced above by writing $\forall[U] \varphi$ for the result of the same procedure, exempting $U$ from being replaced by a variable and bound by a

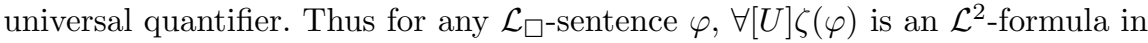
which all relation symbols apart from $U$ are interpreted, and no variable apart from $\vartheta$ is free.

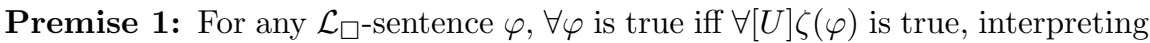
$U$ as being a world and $\vartheta$ as the actual world.

Note that in the version of the standard translation used in this premise, the necessity operator is translated as a universal quantifier restricted to $U$, and first-order quantifiers are unchanged. In neither case is the relevant quantifier relativized to a condition formulated in terms of the variable $\vartheta$ representing the world of evaluation, so Premise 1 is where the assumptions of the necessity of what there is and what is necessary comes into the argument.

To state the second premise of the argument, say that an $\mathcal{L}^{2}$-theory is satisfiable on the intended interpretation if it is true on some interpretation of the non-logical constants, keeping the unrestricted interpretation of first- and second-order quantifiers. Say that such a such a theory is satisfiable on a set 
if it has a model in the standard set-theoretic semantics, according to which first-order quantifiers are interpreted as ranging over the members of a given set $A, n$-ary second-order quantifiers are interpreted as ranging over sets of $n$-tuples on $A$, and non-logical constants are interpreted accordingly.

Premise 2: Every $\mathcal{L}^{2}$-theory satisfiable on the intended interpretation is satisfiable on a set.

Using these two premises, the argument runs as follows: By definition of $\Lambda_{\mathrm{MU}}$, all members of $\left\{\forall \varphi: \varphi \in \Lambda_{\mathrm{MU}}\right\} \cup\left\{\neg \forall \varphi: \varphi \notin \Lambda_{\mathrm{MU}}\right\}$ are true, so by Premise 1 , all members of the $\mathcal{L}^{2}$-theory $\Gamma=\left\{\forall[U] \zeta(\varphi): \varphi \in \Lambda_{\mathrm{MU}}\right\} \cup\left\{\neg \forall[U] \zeta(\varphi): \varphi \notin \Lambda_{\mathrm{MU}}\right\}$ are true, interpreting $U$ as being a world and $\vartheta$ as the actual world. Thus $\Gamma$ is satisfiable on the intended interpretation, and so by Premise 2 satisfiable on a set. In particular, there is a model $\mathfrak{A}=\langle D, e\rangle$ and assignment function $a$ such that $D$ is some cardinal $\kappa, e(U)$ is a cardinal $\lambda \leq \kappa, a(\vartheta) \in \lambda$, and each member of $\Gamma$ is true relative to $\mathfrak{A}$ and $a$. To establish $\Lambda_{\mathrm{MU}}=\Lambda_{\kappa, \lambda}$, it now suffices to prove

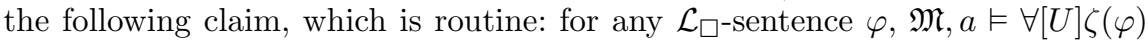
iff $\varphi$ is valid on $\langle\kappa, \lambda\rangle$.

It should be noted that Williamson's argument only applies to $\Lambda_{\mathrm{MU}}$. To turn it into an argument for the claim that $\vDash_{\mathrm{MU}}=\vDash_{\kappa, \lambda}$ for some cardinals $\kappa$ and $\lambda$, one could strengthen Premise 2 to apply to sentences of an extension of $\mathcal{L}^{2}$ which allows for suitably infinitary conjunctions. Although the following focuses on Williamson's original argument concerning $\Lambda_{\mathrm{MU}}$, much of it can be adapted to the case of $\vDash_{\mathrm{MU}}$.

\subsection{Premise 2: Kreisel's Principle}

A version of Premise 2 is discussed in Kreisel (1967), and investigated formally in Shapiro (1987), who calls it Kreisel's Principle. Shapiro formalizes Kreisel's Principle in the context of second-order ZFC. Williamson notes that Shapiro proves it to have a standard model satisfying second-order ZFC, assuming that there are $\aleph_{2}$ inaccessible cardinals. However, such a semantic consistency proof does not establish the truth of the premise. Williamson also notes that Shapiro derives in second-order ZFC that Kreisel's Principle is equivalent to a certain reflection principle in second-order set theory. Such reflection principles have played an important role in modern set theory, and it has been suggested that adopting them can be justified on the basis of the iterative conception of set. However, some variants of such reflection principles have turned out to be inconsistent, and consequently, the status of the (as far as we know) consistent reflection principles is disputed; see, e.g., Koellner (2009) for a recent overview.

Furthermore, it is not even clear that Shapiro's results apply to Premise 2 as used in Williamson's argument, since Shapiro (1987, p. 311) interprets all quantifiers to be restricted to pure sets, whereas Williamson appeals to an unrestricted reading of quantifiers. It is conceivable that Kreisel's Principle holds on pure sets without holding in general, i.e., that every second-order theory satisfiable on the intended interpretation restricted to pure sets is satisfiable on a pure set even though there is some second-order theory satisfiable on the unrestricted intended interpretation which is not satisfiable on any pure (or even impure) set. Further assumptions of (impure) set theory are needed to show that this is not the case. Alternatively, one might directly endorse the relevant 
unrestricted version of Kreisel's Principle, which might turn out to be equivalent to a suitable reflection principle in impure set theory. Although reflection principles are usually discussed in the context of pure set theory, Burgess (2004) introduces an impure set theory containing such a principle as one of its axioms.

\subsection{Premise 1: Worlds in Higher-Order Logic}

Premise 1 captures the assumption that on a suitable understanding of possible worlds, necessity is equivalent to being true in all possible worlds. This won't be questioned in the following. But Premise 1 also contains the assumption that possible worlds can be taken to be individuals, since $\zeta$ replaces $\square$ by a restricted universal first-order quantifier. Maybe they can be so understood according to the modal realism of Lewis (1986), but Williamson (2013, p. 17) explicitly rejects this view.

In line with a view which is perhaps more popular than modal realism (see, e.g., Stalnaker (1976) and Fine (1977)), Williamson's treatment of possible world semantics in (Williamson, 2013, section 3.3) identifies possible worlds with possible propositions which are maximal in the sense of strictly entailing each proposition or its negation. Furthermore, in (Williamson, 2013, chapter 5), propositional quantification is treated as nullary second-order quantification, i.e., quantification into sentence position. It would therefore be natural for Williamson to treat quantification over worlds as restricted quantification into sentence position. $\mathrm{He}$ does not explicitly adopt this position in (Williamson, 2013, section 5.7), where he gives a homophonic interpretation of higher-order modal logic. Rather, he leaves the understanding of worlds open by introducing a primitive type for worlds.

In the following, an argument will be sketched which shows that no matter how worlds are understood, Premise 1 can be adapted without loss of plausibility. For generality, $\mathcal{L}^{2}$ will be extended by a type $w$ for worlds, the interpretation of which will be left open. E.g., quantifiers binding variables of type $w$ might be interpreted as propositional quantifiers restricted to propositions which are maximal in the sense described above. To define the syntax of this extended language $\mathcal{L}^{2 w}$, let the type of a relational symbol be given by a finite sequence of es and $w \mathrm{~s}$, indicating that the symbol combines with a corresponding string of individual and world variables to form an atomic predication. E.g., $\langle e, e, w\rangle$ is the type of a relation symbol $R$ which can be combined with two first-order variables $x$ and $y$ and a world variable $\chi$ to form an atomic predication $R x y \chi$. Based on a countably infinite supply of individual variables, world variables, as well as variables and constants of each relational type, which can be combined in the way just illustrated to form atomic predications, recursively define the formulas of $\mathcal{L}^{2 w}$ using Boolean operations as well as quantifiers binding firstorder, world, and relational variables. However worlds are understood, worlds $\chi$ and $\nu$ are taken to be identical if they are indistinguishable in the sense that $\forall X(X \chi \leftrightarrow X \nu)$, where $X$ is a variable of type $\langle w\rangle$.

To adapt Premise 1 to $\mathcal{L}^{2 w}$, let $\xi$ be a version of the standard translation which differs from $\zeta$ in using a world variable $\theta$ in place of the first-order variable $\vartheta$, and translating $\square$ using an unrestricted universal world-quantifier binding $\theta$ :

$\xi(\square \varphi):=\forall \theta \xi(\varphi)$ 
Of course, each $n$-ary relation symbol must now also be translated using an $n+1$-ary relation symbol of type $\langle e, \ldots, e, w\rangle$. The required premise is then:

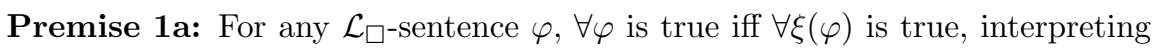
$\theta$ as the actual world.

Grant the truth of this premise for the sake of the argument - how to substantiate it will depend on the understanding of worlds. $\forall \xi(\varphi)$ is in general a sentence of $\mathcal{L}^{2 w}$ rather than $\mathcal{L}^{2}$, so the original argument using Premise 2 (Kreisel's Principle) must be modified. The new argument distinguishes two cases: there being at least as many individuals as worlds, and there being more worlds than individuals.

Since set theory need not be applicable to worlds, how should this talk of "at least as many" and "more" be understood? There being at least as many individuals as worlds should be understood as there being an injective functional relation between worlds and individuals, and there being more words than individuals should be understood as there being no such relation. Here, quantification over relations between worlds and individuals should be understood as quantification binding variables of type $\langle w, e\rangle$, i.e., variables which combine with an expression of type $w$ (denoting a world) and an expression of type $e$ (denoting an individual) to form a sentence. For readability, the argument is written in English using phrases such as "relation between worlds and individuals", rather than a formal higher-order language, in which it would be expressed more properly.

Case 1: There are at least as many individuals as worlds; i.e., there is an injection $i$ from worlds to individuals. For this case, the argument relies on the following premise:

Premise 1b: If there is an injection $i$ from worlds to individuals, then for

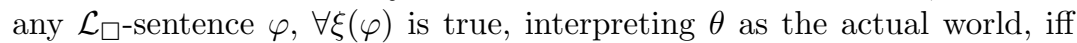
$\forall[U] \zeta(\varphi)$ is true, interpreting $U$ as the image of $i$ and $\vartheta$ as the image of the actual world under $i$.

This premise is motivated by the kind of unrestricted comprehension principle endorsed in Williamson (2013, p. 227): with it, any sequence of relations witnessing the falsity of $\forall \xi(\varphi)$ can be turned, via $i$, into a counterexample of $\forall[U] \zeta(\varphi)$, and vice versa. Premises $1 \mathrm{a}$ and $1 \mathrm{~b}$ entail:

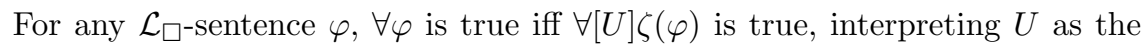
image of $i$ and $\vartheta$ as the image of the actual world under $i$.

This claim can take the place of Premise 1 in the original argument.

Case 2: There are more worlds than individuals. (The following would also apply to the case in which there are as many worlds as individuals, but this is already covered by Case 1.) In this case, it will be shown that $\Lambda_{\leq}=\Lambda_{\mathrm{MU}}$, first showing $\subseteq$ and then $\supseteq$.

Assume first that $\varphi \in \Lambda_{\leq}$. Then by Theorem $23, \varphi$ is derivable in $\mathrm{LPC}^{\infty}+\mathrm{S} 5$. With Premise 1c it follows that $\varphi \in \Lambda_{\mathrm{MU}}$, as required:

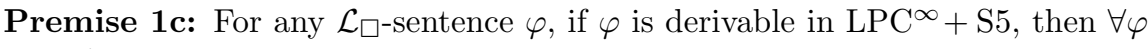
is true. 
A natural way of substantiating this premise is by an inductive argument over length of derivation in (a suitably modified version of) $\mathrm{LPC}^{\infty}+\mathrm{S} 5$ along the lines of the argument in (Williamson, 2013, section 3.3) for the propositional case.

For the other direction, it is helpful to establish an auxiliary claim for any

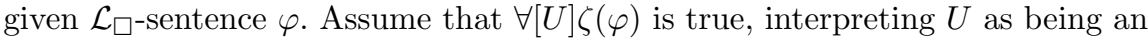
individual (and $\vartheta$ as some particular individual - it does not matter which). On this interpretation, $\neg \forall[U] \zeta(\neg \eta)$ is true, where $\eta=\forall x \diamond \forall y(I y \leftrightarrow x=y)$ (interpret $\rho(I)$ as identity). By Premise 2, $\{\forall[U] \zeta(\varphi), \neg \forall[U] \zeta(\neg \eta)\}$ is therefore satisfiable on a cardinal $\kappa$, interpreting $U$ as a cardinal $\lambda \leq \kappa$. Thus $\varphi$ is valid on $\langle\kappa, \lambda\rangle$, but $\neg \eta$ is not. Since $\neg \eta$ is not valid on $\langle\kappa, \lambda\rangle, \kappa=\lambda$, and therefore $\varphi \in \Lambda_{\leq}$.

Contraposing this line of reasoning, if $\varphi \notin \Lambda_{\leq}$, then $\forall[U] \zeta(\varphi)$ is false, interpreting $U$ as being an individual and $\vartheta$ as some individual. With Premise $1 \mathrm{~d}$ it follows that $\forall \xi(\varphi)$ is false, interpreting $\theta$ as the actual world, and so, via Premise 1a, that $\varphi \notin \Lambda_{\mathrm{MU}}$, as required:

Premise 1d: If there are more worlds than individuals, then for any $\mathcal{L}_{\square}$ sentence $\varphi$, if $\forall[U] \zeta(\varphi)$ is false, interpreting $U$ as being an individual and $\vartheta$ as some individual, then $\forall \xi(\varphi)$ is false, interpreting $\theta$ as the actual world.

This premise can be motivated by adapting the proof idea of Lemma 2: Since there are more worlds than individuals, there is a surjection $s$ from worlds to individuals, mapping the actual world to the individual used to interpret $\vartheta$. As in the argument for Premise $1 \mathrm{~b}$, given a sequence of relations which witnesses the falsity of $\forall[U] \zeta(\varphi)$, the unrestricted comprehension principle allows us to construct, via $s$, a sequence of relations witnessing the falsity of $\forall \xi(\varphi)$, interpreting $\theta$ as the actual world.

\section{References}

Jon Barwise. Some eastern two cardinal theorems. In Robert E. Butts and Jaakko Hintikka, editors, Logic, Foundations of Mathematics, and Computability Theory, pages 11-31. Dordrecht: D. Reidel, 1977.

Kenneth A. Bowen. Model Theory for Modal Logic: Kripke Models for Modal Predicate Calculi. Dordrecht: D. Reidel, 1979.

John P. Burgess. E pluribus unum: plural logic and set theory. Philosophia Mathematica, 12:193-221, 2004.

C. C. Chang. A note on the two cardinal problem. Proceedings of the American Mathematical Society, 16:1148-1155, 1965.

C. C. Chang and H. Jerome Keisler. Model Theory. Amsterdam: North-Holland, 3rd edition, 1990.

Keith J. Devlin. Constructibility. Berlin: Springer-Verlag, 1984.

Kit Fine. Postscript to Worlds, Times and Selves (with A. N. Prior). London: Duckworth, 1977. 
Harvey M. Friedman. A complete theory of everything: Satisfiability in the universal domain. http://www.math.ohio-state.edu/ friedman/, 1999.

Peter Fritz. Modal ontology and generalized quantifiers. Journal of Philosophical Logic, 42:643-678, 2013.

G. Fuhrken. Skolem-type normal forms for first-order languages with a generalized quantifier. Fundamenta Mathematicae, 54:291-302, 1964.

G. E. Hughes and M. J. Cresswell. A New Introduction to Modal Logic. London: Routledge, 1996.

R. Björn Jensen. The fine structure of the constructible hierarchy. Annals of Mathematical Logic, 4:229-308, 1972.

H. Jerome Keisler. First order properties of pairs of cardinals. Bulletin of the American Mathematical Society, 72:141-144, 1966.

Peter Koellner. On reflection principles. Annals of Pure and Applied Logic, 157: 206-219, 2009.

Georg Kreisel. Informal rigour and completeness proofs. In Imre Lakatos, editor, Problems in the Philosophy of Mathematics, pages 138-186. Amsterdam: North-Holland, 1967.

Saul A. Kripke. A completeness theorem in modal logic. Journal of Symbolic Logic, 24:1-14, 1959.

David Lewis. On the Plurality of Worlds. Oxford: Basil Blackwell, 1986.

William Mitchell. Aronszajn trees and the independence of the transfer property. Annals of Mathematical Logic, 5:21-46, 1972.

Michael Morley and Robert Vaught. Homogeneous universal models. Mathematica Scandinavica, 11:37-57, 1962.

Agustín Rayo and Timothy Williamson. A completeness theorem for unrestricted first-order languages. In JC Beall, editor, Liars and Heaps: New Essays on Paradox, pages 331-356. Oxford: Oxford University Press, 2003.

James H. Schmerl. An axiomatization for a class of two-cardinal models. The Journal of Symbolic Logic, 42:174-178, 1977.

Stewart Shapiro. Principles of reflection and second-order logic. Journal of Philosophical Logic, 16:309-333, 1987.

Saharon Shelah. On successors of singular cardinals. In Maurice Boffa, Dirk van Dalen, and Kenneth McAloon, editors, Logic Colloquium 78, pages 357-380. Amsterdam: North-Holland, 1979.

Saharon Shelah. The pair $\left(\aleph_{n}, \aleph_{0}\right)$ may fail $\aleph_{0}$-compactness. In Matthias Baaz, Sy-David Friedman, and Jan Krajíček, editors, Logic Colloquium '01, pages 402-433. A K Peters/CRC Press, 2005. 
Jack Silver. The independence of Kurepa's conjecture and two-cardinal conjectures in model theory. In Dana S. Scott, editor, Axiomatic Set Theory. Proceedings of Symposia in Pure Mathematics, Volume XIII, Part I, pages 383-390. Providence: American Mathematical Society, 1971.

Robert Stalnaker. Possible worlds. Nô̂s, 10:65-75, 1976.

Alfred Tarski. On the concept of following logically. History and Philosophy of Logic, 23:155-196, 2002 [1936]. Originally published in Polish and German in 1936.

R. L. Vaught. The completeness of logic with the added quantifier "there are uncountably many". Fundamenta Mathematicae, 54:303-304, 1964.

R. L. Vaught. A Löwenheim-Skolem theorem for cardinals for apart. In J. W. Addison, L. Henkin, and A. Tarski, editors, The Theory of Models, pages 390-401. Amsterdam: North-Holland, 1965a.

R. L. Vaught. The Löwenheim-Skolem theorem. In Yehoshua Bar-Hillel, editor, Logic, Methodology and Philosophy of Science, pages 81-89. Amsterdam: North-Holland, 1965b.

Timothy Williamson. Existence and contingency. Proceedings of the Aristotelian Society, 100:321-343, 2000a.

Timothy Williamson. The necessary framework of objects. Topoi, 19:201-208, 2000b.

Timothy Williamson. Everything. Philosophical Perspectives, 17:415-465, 2003.

Timothy Williamson. Modal Logic as Metaphysics. Oxford: Oxford University Press, 2013. 